\title{
Sodium channel blockers in the management of long QT syndrome: a system review and meta-analysis
}

\author{
Ying Yang ${ }^{1}$, Tingting $\mathrm{Lv}^{1}$, Siyuan $\mathrm{Li}^{1}$, and Ping Zhang ${ }^{1}$ \\ ${ }^{1}$ Tsinghua University
}

July 6, 2021

\begin{abstract}
Background: $\beta$-blockers are first-line therapy in patients with long QT syndrome (LQTS). However, $\beta$-blockers had genotype dependent efficacy (LQT1>LQT2>LQT3). Sodium channel blockers have been recommended as add-on therapy for LQT3 patients. However, the effect of sodium channel blockers in all LQT patients remains unknown. Methods: We conducted a systematic electronic search of PubMed, Embase and the Cochrane Library. Fixed effects model was used to assess the effect of sodium channel blockers on QTc, cardiac events (CEs), and the proportion of QTc[?]500 ms and QTc[?]460 ms in LQTS patients. Results: Pooled analysis of 14 studies with 213 LQTS patients showed that sodium channel blockers significantly shortened QTc by nearly $50 \mathrm{~ms}$ (MD $-49.43,95 \% \mathrm{CI}-57.80$ to $-41.05, \mathrm{P}<0.001)$, reduced the incidence of CEs (OR $0.12,95 \% \mathrm{CI} 0.04$ to $0.32, \mathrm{P}<0.001)$ and the proportion of QTc[?]500 ms (OR 0.15, 95\% CI 0.09 to $0.26, \mathrm{P}<0.001)$, and increased the proportion of QTc[?]460 ms (OR 18.00, 95\%CI 7.49 to 43.26, P<0.001). Sodium channel blockers significantly shortened QTc both in LQT3 and non-LQT3 patients, while the QTc shortening effect in LQT3 was superior to that in non-LQT3 (57.39 ms vs. $36.61 \mathrm{~ms}$ ). Mexiletine, flecainide, and ranolazine all significantly shortened QTc, and the QTc shortening effect by mexiletine was the best (60.70 ms vs. $49.08 \mathrm{~ms}$ vs. $50.10 \mathrm{~ms}$ ). Conclusions: sodium channel blockers can be useful both in LQT3 and non-LQT3 patients. Mexiletine, flecainide and ranolazine significantly shortened QTc in LQTS patients, and the QTc shortening effect by mexiletine was the best.
\end{abstract}

\section{Hosted file}

Sodium channel blockers in the management of long QT syndrome a system review and meta-analysis.doc available at https://authorea.com/users/424026/articles/529207-sodium-channel-blockers-inthe-management-of-long-qt-syndrome-a-system-review-and-meta-analysis 